\title{
THE ISOPERIMETRIC PROBLEM ON PLANES WITH DENSITY
}

\author{
COLIN CARROLL, ADAM JACOB, CONOR QUINN and ROBIN WALTERS ${ }^{\bowtie}$
}

\author{
(Received 18 September 2007)
}

\begin{abstract}
We discuss the isoperimetric problem in planes with density. In particular, we examine planes with generalized curvature zero. We solve the isoperimetric problem on the plane with density $e^{x}$, as well as on the plane with density $r^{p}$ for $p<0$. The Appendix provides a proof by Robert Bryant that the Gauss plane has a unique closed geodesic.
\end{abstract}

2000 Mathematics subject classification: 53C42.

Keywords and phrases: isoperimetric problem, planes with density, Gauss plane.

\section{Introduction}

The isoperimetric problem on a Riemannian surface seeks a region of least perimeter enclosing a given area. In general, we can study this problem on a surface where a continuous positive function, called the density, is used to weight both area and perimeter. Note that defining such a density is not equivalent to scaling the metric by a factor $\lambda$, because area and perimeter would be scaled by different powers of $\lambda$.

We consider the particular case of the Euclidean plane with a density function $e^{\varphi}$. We are especially interested in what conclusions can be drawn when the generalized Gauss curvature of the plane is zero, which occurs when $\varphi$ is harmonic. For the plane with density $r^{p}$, Section 4 shows that isoperimetric regions exist for $p \geq 0$ or $p<-2$ but not for $-2 \leq p<0$, when any area may be enclosed by arbitrarily small perimeter. For the plane with density $e^{x}$, given $A>0$, the infimum perimeter is $A$ but cannot be attained (Proposition 4.9). Section 5 studies the ' $g$-plane' with density $\exp \left(x^{2}-y^{2}\right)$.

In Section 2 we cite the definition of generalized Gauss curvature and the generalized Gauss-Bonnet formula for surfaces with density from Corwin et al. [3]. We conclude this section with some general results about geodesics in planes with generalized curvature zero.

Our thanks are due to the National Science Foundation for grants to Morgan and SMALL, and Williams College.

(c) 2008 Australian Mathematical Society 0004-9727/08 \$A2.00+0.00 
In Section 3 we consider surfaces with an area density function $e^{\beta}$ that weights area but leaves perimeter unaffected, and we provide generalizations of the curvature of a curve and Gauss-Bonnet. Proposition 3.4 explains that any plane with density can be viewed under a conformal change of variables as a surface with area density. If the generalized curvature of the plane is zero, then the surface is flat and has Euclidean coordinates. This observation leads to many important conclusions about planes with generalized Gauss curvature zero. For nonconstant density, there does not exist a geodesic between every two points and there are no compact isoperimetric regions (Proposition 3.5 and Corollary 3.7).

In Section 4 we solve the isoperimetric problem on planes with density $e^{x}$ and $r^{p}$, $p<0$. For a plane with density $r^{p}, p>0$, we note that circles about the origin are unstable and conjecture that minimizers are off-centre, convex discs.

In Section 5 we explore the plane with density $\exp \left(x^{2}-y^{2}\right)$, which we call the g-plane. We are interested in $\mathcal{g}$ because of its relationship to the Gauss plane of density $\exp \left(-x^{2}-y^{2}\right)$ and because it has generalized curvature zero. We prove some results about the behaviour of the geodesics of $\mathcal{g}$. For the isoperimetric problem, we conjecture that, similar to the plane with density $e^{x}$, the infimum perimeter for given area is not attained.

In Section Appendix A we conclude with a proof by Robert Bryant that the Gauss plane has a unique closed geodesic, a circle.

\section{Manifolds with density}

We consider Riemannian surfaces endowed with a positive density $e^{\varphi}$ used to weight both perimeter and area. In terms of Riemannian perimeter and area $\left(d P_{0}\right.$ and $d A_{0}$ ), weighted perimeter and area satisfy

$$
\begin{aligned}
& d P=e^{\varphi} d P_{0}, \\
& d A=e^{\varphi} d A_{0} .
\end{aligned}
$$

Note that such a metric is not equivalent to scaling the perimeter and area by some factor $\lambda$, since perimeter and area would scale by different powers of $\lambda$. See Morgan [7] for more on manifolds with density.

The tools for studying surfaces with density from Corwin et al. [3] include a formula for the generalized (Gauss) curvature

$$
G_{\varphi}=G-\Delta \varphi
$$

where $G$ is the classical Gauss curvature. The curvature of a curve is given by

$$
\kappa_{\varphi}=\kappa-\frac{d \varphi}{d n}
$$


where $\kappa$ is the classical curvature. As in the classical case, $\kappa_{\varphi}$ has the interpretation of $d P / d A$, and for the boundary of an isoperimetric region, $\kappa_{\varphi}$ is constant. Finally, the Gauss-Bonnet formula generalizes to surfaces with density:

$$
\int_{R} G_{\varphi} d A_{0}+\int_{\partial R} \kappa_{\varphi} d P_{0}+\sum\left(\pi-\alpha_{i}\right)=2 \pi .
$$

We begin with two results about geodesics.

PROPOSITION 2.1. Let $\Pi$ be a plane with density $e^{\varphi}$ with generalized curvature $G_{\varphi}=0$. Then there are no closed geodesics in $\Pi$.

PROOF. Let $\gamma$ be a closed curve of constant curvature in $\Pi$. Since $\Pi$ is a plane, the bounded region is a disc, so Gauss-Bonnet applies:

$$
2 \pi=\int_{\gamma} \kappa_{\varphi} d P_{0}=\kappa_{\varphi} \int_{\gamma} d P_{0}=\kappa_{\varphi} P_{0} .
$$

This equation cannot hold if $\kappa_{\varphi}=0$, so no geodesic can be closed.

PROPOSITION 2.2. Given a plane with density that has nonpositive generalized curvature, if there exists a geodesic $\gamma$ between two points, then it is unique.

PROOF. Let $\gamma$ and $\gamma^{\prime}$ be distinct geodesics between two points in the plane. Begin at one point, which we shall call $a$, and follow $\gamma$ until it first crosses $\gamma^{\prime}$ (which may not be until the other point) and call that intersection point $b$. Now since we are in the plane and the bounded region is a disc, we can apply Gauss-Bonnet to the region bounded by $\gamma$ and $\gamma^{\prime}$, with $\alpha_{1}$ and $\alpha_{2}$ the angles formed where the two geodesics meet. Thus,

$$
\int_{R} G_{\varphi}+\int_{\gamma} \kappa_{\varphi}+\int_{\gamma^{\prime}} \kappa_{\varphi}+\sum\left(\pi-\alpha_{i}\right)=2 \pi .
$$

Because $\gamma$ and $\gamma^{\prime}$ are both geodesics, the $\int \kappa_{\varphi}$ terms vanish, so that

$$
\alpha_{1}+\alpha_{2}=\int_{R} G_{\varphi}
$$

Since $G_{\varphi} \leq 0, \alpha_{1}=\alpha_{2}=0$, and the geodesics must coincide.

\section{Manifolds with area density}

We now consider Riemannian surfaces with a positive area density $e^{\beta}$ that weights area but not perimeter. Proposition 3.4 shows that a Riemannian surface with density is equivalent to a Riemannian surface with area density. Proposition 3.5 shows that geodesics do not exist between every two points on a plane with nonconstant density and $G_{\varphi}=0$. Corollary 3.7 shows that in a plane $\Pi$ with density $e^{\varphi}$ and $G_{\varphi}=0$, there are no compact isoperimetric regions. 
DEFINITION 3.1. Let $\gamma$ be a smooth curve in a surface with area density $e^{\beta}$. Then the curvature $\kappa_{\beta}$ at any point along $\gamma$ is defined to be

$$
\kappa \beta=\kappa e^{-\beta},
$$

where $\kappa$ is the classical curvature.

Note that $\kappa_{\beta} \geq 0$ if and only if $\kappa \geq 0$.

This definition is justified by the following proposition.

Proposition 3.2. Infinitesimally, $\kappa_{\beta}=d P / d A$.

In particular, for the boundary of an isoperimetric region, $\kappa_{\beta}$ is constant.

PROOF. Length is unaffected by the area density, so the standard first variation formula [4, p. 6] holds:

$$
\frac{d P}{d t}=\int \kappa \cdot v d P
$$

However, area is affected, and as a result $d A / d t=\int v \cdot e^{\beta} d P$. The result follows.

Proposition 3.3 (Generalized Gauss-Bonnet). Given a piecewise smooth curve enclosing a topological disk $R$ in a Riemannian surface with area density $e^{\beta}$ and inward pointing unit normal $\mathbf{n}$,

$$
\int_{R} G d A_{0}+\int_{\partial R} \kappa_{\beta} e^{\beta} d P_{0}+\sum\left(\pi-\alpha_{i}\right)=2 \pi .
$$

PROOF. The proof follows from direct substitution into the Riemannian GaussBonnet formula.

PROPOSITION 3.4. Every surface with density $e^{\varphi}$ is conformally equivalent to a (not necessarily complete) surface with area density $e^{\beta}=e^{-\varphi}$. In particular, every plane with generalized curvature zero is conformally equivalent to a flat surface with area density; its development over the Euclidean plane yields Euclidean coordinates.

PROOF. Considering length alone, a density function on the surface is equivalent to a conformal change of metric $d P=e^{\varphi} d P_{0}$, yielding $d A=e^{2 \varphi} d A_{0}$. Adding area density $e^{\beta}=e^{-\varphi}$ yields $d A=e^{\varphi} d A_{0}$, as desired.

Suppose that the surface with density has Gauss curvature $G$ and generalized curvature $G_{\varphi}=G-\Delta \varphi$. The conformal change of metric yields Gauss curvature

$$
G^{\prime}=e^{-2 \varphi}(G-\Delta \varphi)=e^{-2 \varphi} G_{\varphi} ;
$$

see [1, Theorem 1.159] and [8, 3.2]. Hence, if $G_{\varphi}=0, G^{\prime}=0$, and the plane with area density can be developed over the Euclidean plane (as a Riemann surface). 
REMARK. Proposition 3.4 implies Propositions 2.1 and 2.2.

PROPOSITION 3.5. Given a plane with nonconstant density $e^{\varphi}$ and generalized curvature zero, there does not exist a geodesic between every pair of points.

ProOf. Assume that a geodesic exists between every pair of points. By Proposition 3.4 there exists a conformal mapping that converts the plane to a flat Riemannian manifold $M$ with Euclidean coordinates. Under this mapping geodesics of the plane become straight lines, and, because there exists a geodesic between every pair of points, $M$ must be a convex subset of the plane. If $M$ is not the whole plane, then by the Riemann mapping theorem $M$ maps conformally to the unit disk, and is bounded. But $\varphi$ is harmonic and nonconstant, so $M$ bounded contradicts Liouville's theorem, implying that $M$ is the whole Euclidean plane. Now we show that any conformal automorphism of the complex plane is linear, which would imply that the density is constant, a contradiction that proves the proposition.

A conformal automorphism of the complex plane is a map from the Riemann sphere to itself with a singularity at infinity. If this singularity is essential, it takes on almost all values infinitely often (by Picard's theorem), which would contradict injectivity. Thus the singularity is not essential. So the conformal map is a linear fractional transformation, and because it takes infinity to infinity it must be of the form $w=a z+b$ ( $z$ cannot be in the denominator) and the map is linear. Therefore the density is constant, which implies a contradiction, so there does not exist a geodesic between every pair of points.

PROPOSITION 3.6. Let $f$ be a nonconstant subharmonic function on an open domain $D$ in $\mathbb{R}^{2}$. Consider the class of subsets $S$ of $D$ which are translates of a fixed compact subset $S_{0}$ of $D$. Let $g(S)$ denote the integral of $f$ over $S$. Then $g$ cannot have a local maximum at $S_{0}$.

PROOF. Define a function $h$ in a neighbourhood of 0 where $h(x)$ is the average of $f$ over $S_{0}+x$. $h$ is well defined because the integral over a region translated by some $x$ always exists in the open domain $D$. As an integral average of nonconstant subharmonic functions, $h$ is nonconstant subharmonic. Hence $h$ cannot have a local maximum at 0 , so $g$ cannot have a local maximum at $S_{0}$.

COROLlary 3.7. In a plane $\Pi$ with nonconstant density $e^{\varphi}$ such that $G_{\varphi}=0$, an isoperimetric region has no compact components.

PROOF. Suppose that an isoperimetric region has a compact component $R$. By Proposition 3.4, a conformal change of metric yields Euclidean coordinates with area density $e^{\beta}=e^{-\varphi}$. Since $\varphi$ remains harmonic [2, Theorem 8.4], $e^{\beta}$ is subharmonic. By Proposition 3.6, any compact isoperimetric region cannot be a local maximum for area. By the remark after Definition 3.1 and Proposition 3.2, the outer boundary of $R$ must be strictly convex with constant curvature. Move $R$ slightly to increase area. Now 
replace some arc on the boundary with a chord to restore area and lower perimeter, thus contradicting isoperimetry.

\section{Planes with density $r^{p}$ and $e^{x}$}

In this section we explore the isoperimetric problem in planes with density $e^{x}$ and $r^{p}$, where $p$ is any real number. Although $\Delta \log r^{p}=0$, we cannot assume all the previous results about planes with generalized curvature zero for these examples, since at the origin $r^{p}=0$ and $\log r^{p}$ is undefined.

Proposition 4.1. Circles about the origin in the plane with density $r^{-1}$ are geodesics.

PROOF. By symmetry, a circle about the origin has constant generalized curvature. As the circles grow, the enclosed weighted area $A$ increases, while the weighted perimeter $P$ remains $2 \pi r r^{-1}=2 \pi$. Hence the generalized curvature, which has the geometric interpretation $d P / d A$, vanishes, and the circles are geodesics.

This can be easily observed by looking at Euclidean coordinates. Here a circle of radius $r$ is mapped to the line $x=\log (r)$ and thus has a boundary of zero classical curvature. By Definition 3.1 all circles have generalized curvature equal to zero.

PROPOSITION 4.2. Given a plane $\Pi$ with density $r^{p},-2 \leq p<0$, there does not exist a solution to the isoperimetric problem. Indeed, regions of any area A can be enclosed by an arbitrarily small perimeter.

PROOF. In $\Pi$, the radius of a circle about the origin is $2 \pi r^{p+1}$. If $-2 \leq p<-1$, as $r$ approaches infinity the perimeter of the circle goes to zero. The area outside of the circle is equal to

$$
\int_{0}^{2 \pi} \int_{r}^{\infty} r^{p+1} d r d \theta=\infty .
$$

So given any $\varepsilon>0$, we can construct two circles about the origin with large enough radii such that the combined perimeter of both is less than $\varepsilon$. Let $A_{0}$ be the area in between the two circles. If $A_{0}<A$, because there is infinite area outside of the second circle, we can increase the radius of that circle, decreasing perimeter and increasing area to $A$. If $A_{0}>A$, then we increase the radius of the first circle, decreasing both perimeter and area to reach area $A$.

Now suppose that $p=-1$. Euclidean coordinates are produced by the mapping $w=\log (z)$, with $|d w|=\left|z^{-1} d z\right|$ and area density $|z|=\left|e^{w}\right|=e^{x}$ (with $x=\operatorname{Re} w$ ). The image of $\Pi$ under this map is a strip of height $2 \pi$ with the top and bottom identified. The origin maps to negative infinity, and circles of radius $r$ map to vertical lines $(x=\log r)$. Given $\varepsilon>0$, consider a circle with perimeter less than $\varepsilon$. This circle can be slid left or right to attain any desired area.

If $-1<p<0$ then the image of $\Pi$ under the mapping $w=z^{p+1} /(p+1)$ is a sector in the plane with identified sides and vertex angle $2 \pi(p+1)$. The area density in 
Euclidean coordinates is given by $|z|^{-p}=|w|^{-p /(p+1)}$, which gives zero area density at the origin and approaches infinity as $r$ goes to infinity. Once again given any $\varepsilon>0$, in Euclidean coordinates create a circle with perimeter $P<\varepsilon$ and area $A_{0}$. If $A<A_{0}$ then we can contract the circle, decreasing the perimeter until $A_{0}=A$. If $A>A_{0}$, then the circle can be slid outward to attain any desired area $A$ by the intermediate value property.

PROPOSITION 4.3. In the plane $\Pi$ with density $r^{p}$ where $p<-2$, a circle about the origin encloses a given area $A$ (on the outside) with the least perimeter.

PROOF. We work in Euclidean coordinates, produced by the mapping $w=z^{p+1}$ / $(p+1)$, with $|d w|=\left|z^{p} d z\right|$ and area density $|z|^{-p}=c|w|^{-q}$, with $q=p /(p+1)$. So $1<q<2$. The image of $\Pi$ is a sector of angle $2 \pi|p+1|$ with bounding rays identified. Since the area density goes to 0 as $|w|$ goes to infinity, components of regions of prescribed area can be moved closer to the origin and shrunk. Therefore there is a bounded sequence of regions approaching the infimum perimeter, and a minimizer exists by standard compactness arguments (see, for example, [5, Sections 5.5 and 9.1]). Any minimizer is bounded by a smooth curve of constant curvature [6, Section 3.10] (see Definition 3.1) and hence is convex. The region has just one component, because if there were two components, one could be moved closer to the origin and shrunk. Likewise the region must contain the origin, so its boundary is a graph $r(\theta)$ in polar coordinates, with $\theta$ ranging from 0 to $L=2 \pi|p+1|$. The area $A_{R}$ is given by

$$
\begin{aligned}
A_{R} & =c \int_{0}^{L} \int_{0}^{r(\theta)} r_{0}^{-p /(p+1)} r_{0} d r_{0} d \theta \\
& =c \int_{0}^{L} \int_{0}^{r(\theta)} r_{0}^{1 /(p+1)} d r_{0} d \theta \\
& =c \frac{p+1}{p+2} \int_{0}^{L}\left[r_{0}^{(p+2) /(p+1)}\right]_{0}^{r(\theta)} d \theta \\
& =c_{2} \int_{0}^{L} r(\theta)^{(p+2) /(p+1)} d \theta
\end{aligned}
$$

where $c_{2}=c(p+1) /(p+2)$.

We now consider a circle $C$ of radius $r_{\text {avg }}$ where $r_{\text {avg }}$ is the average value of the function $r(\theta)$ on $[0, L]$. This circle has perimeter

$$
\begin{aligned}
P_{C} & =L r_{\mathrm{avg}}=\int_{0}^{L} r_{\mathrm{avg}} d \theta=\int_{0}^{L} r(\theta) d \theta=\int_{0}^{L} \sqrt{r(\theta)^{2}} d \theta \\
& <\int_{0}^{L} \sqrt{r(\theta)^{2}+\dot{r}(\theta)^{2}} d \theta=P_{R} .
\end{aligned}
$$


So the perimeter of $C$ is less than that of $R$. To show that $C$ contains greater area, we note from our earlier calculation that

$$
A_{C}=c_{2} \int_{0}^{L}\left(r_{\mathrm{avg}}\right)^{(p+2) /(p+1)} d \theta .
$$

Now let $g(x)=c_{2} x^{(p+2) /(p+1)}$. Then since $0<(p+2) /(p+1)<1$ for $p<-2$, and $c_{2}>0, g(x)$ is strictly concave for $x>0$. Hence,

$$
A_{C}=\int_{0}^{L} g\left(r_{\mathrm{avg}}\right) d \theta>\int_{0}^{L} g(r(\theta)) d \theta=A_{R} .
$$

So $A$ has greater area as well.

PROpOSITION 4.4. Given a plane with density $r^{p}, p \geq 0$, then there exists an isoperimetric region.

Proof. Rosales et al. [9, Theorem 2.6] proved that in Euclidean space endowed with a nondecreasing radial density function $f$, such that $f(|x|) \rightarrow \infty$ as $|x| \rightarrow \infty$, a minimizer exists. We note that although Rosales dealt with manifolds of strictly positive density, the argument admits an isolated point of zero density.

PROPOSITION 4.5. Given a plane with density $r^{p}, p>0$, any isoperimetric region must contain the origin in its interior or its boundary.

PROOF. Suppose that it does not. Then an isoperimetric region exists some distance from the origin with area $A$ and perimeter $P$. By $[6,3.10]$, the boundary of the region must be a closed curve of constant curvature. Therefore, in the Euclidean coordinates (attained through the mapping $w=z^{p+1} /(p+1)$ ), the boundary must be strictly convex. The weighted area density function in Euclidean coordinates is the inverse of the original density function and is radially decreasing. As in the proof of Corollary 3.7, any convex region some finite distance from the origin can be slid closer to the origin into increasing area density, thus increasing the area of the region. By replacing an arc of the boundary with a chord, we can reduce the area of the new region back down to $A$. However, this new region has less perimeter than the original isoperimetric region. Thus it could not have been isoperimetric.

Conjecture 4.6. In the plane $\Pi$ with density $r^{p}, p>0$, for a given area $A$ the isoperimetric region $R$ is an off-centre, convex disk, containing the origin as in Figure 1. It approaches a circle through the origin as p goes to zero and approaches a unit ray as $p$ goes to infinity.

REMARK. We offer the following evidence for Conjecture 4.6. By Proposition 4.4, a minimizer exists. By [6, Section 3.10] it is smooth, except possibly at the origin. The boundary has at most one smooth component; otherwise expanding one and shrinking another by constant normal variations could preserve enclosed area to first order and 


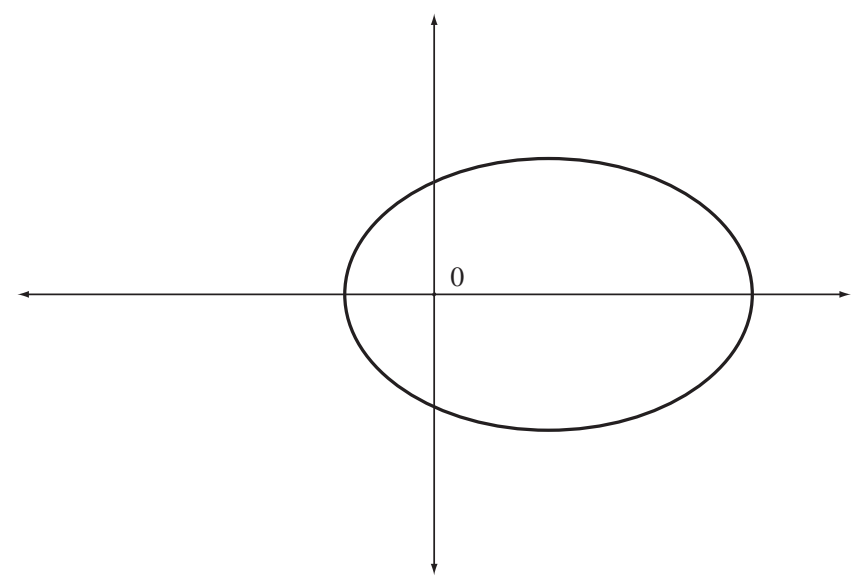

FIGURE 1. The conjectured minimizer for the plane with density $r^{p}, p>0$.

reduce perimeter to second order by the second variation formula [9, Proposition 3.6]. Since a smooth boundary has constant generalized curvature, it probably is convex. By Rosales et al. [9, Theorem 3.10], circles about the origin are stable if and only if the density function is $\log$ convex. Since $r^{p}$ is not $\log$ convex, circles about the origin are not stable. However, though the isoperimetric region $R$ is not a circle, by Proposition 4.5, $R$ must contain the origin. We think that the origin lies in the interior because in Euclidean coordinates the area density blows up at the origin.

In the first limit case, when $p=0$, the minimizer $R$ can be a circle centred about any point in the plane. However, when looking at the limit as $p$ goes to zero, a limit of minimizers must be a circle through the origin. Indeed, as $p$ approaches zero, the dip at the origin of the density function gets smaller and smaller, and for each minimizer it pays for part of the boundary to travel through that dip because passing through the dip lowers perimeter while gaining area.

In the second limit case, as $p$ becomes large, a minimizer must extend beyond the unit circle (to have given area) but probably only slightly beyond.

PROPOSITION 4.7. In the plane with density $e^{x}$, all curves with constant curvature $\kappa_{\varphi}$ are either closed or have infinite (weighted) length.

PROOF. Assume that they do not. Then there exists a curve, denoted $\gamma$, that is not closed but has finite weighted length. $\gamma$ must contain a point $p$ with maximum $x$ value, otherwise $\gamma$ would have infinite weighted length. At $p$ the normal vector $\mathbf{n}=-\mathbf{i}$. Also, $\kappa>0$; hence $\kappa_{\varphi}=\kappa-d \varphi / d n>1$. Hence, everywhere $\kappa=\kappa_{\varphi}+d \varphi / d n$ is bounded below by the positive constant $\kappa_{\varphi}-1$, a contradiction.

COROLlARY 4.8. The plane with density $e^{x}$ contains no isoperimetric region. 


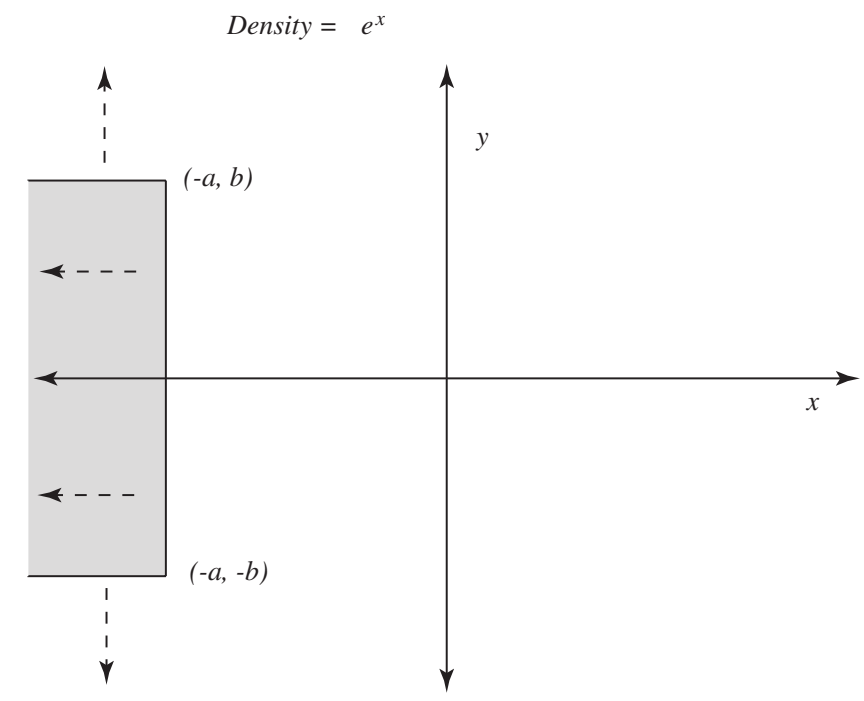

FIGURE 2. A region in the plane with density $e^{x}$ that approaches minimal perimeter for given area.

PROOF. Any isoperimetric region $R$ must have a smooth boundary with constant curvature $\kappa_{\varphi}[6,3.10]$. By Proposition 4.7, $R$ has a compact component, contradicting Corollary 3.7.

Although the plane with density $e^{x}$ has no specific region that realizes the minimal area to perimeter ratio for the plane, the minimal ratio can be approached in the limit of certain noncompact regions (see Figure 2).

Proposition 4.9. For the plane with density $e^{x}$, the infimum of perimeters to enclose a region with area $A$ is $A$. This infimum is not realized by any region.

PROOF. Construct an open rectangle $R$ as in Figure 2, symmetric about the $x$-axis. Simple calculations show that the perimeter and area are given by

$$
\begin{gathered}
P_{R}=e^{-a}(2 b+1), \\
A_{R}=2 b e^{-a} .
\end{gathered}
$$

Given any area $A$, let $a$ be a function of $b$ so that for any $b, A_{R}=A$. Now let $b$ go to infinity. Then $a$ goes to infinity as well, so $e^{-a}$ converges to zero, and the ratio $A_{R} / P_{R}$ goes to one.

Now suppose that there exists a region with perimeter less than or equal to $A$. Looking at all horizontal slices of the plane, we see that the most efficient way to enclose length in the slice is a point with density $e^{p}$ enclosing length $e^{p}$ to the left. The area of the region is given by the integral of all the horizontal length slices $e^{p}$, and the perimeter is given by the integral of $e^{p}$ times a tilt factor greater than or equal to 1 . Since the boundary cannot have a vertical line, the perimeter is greater than $A$. 


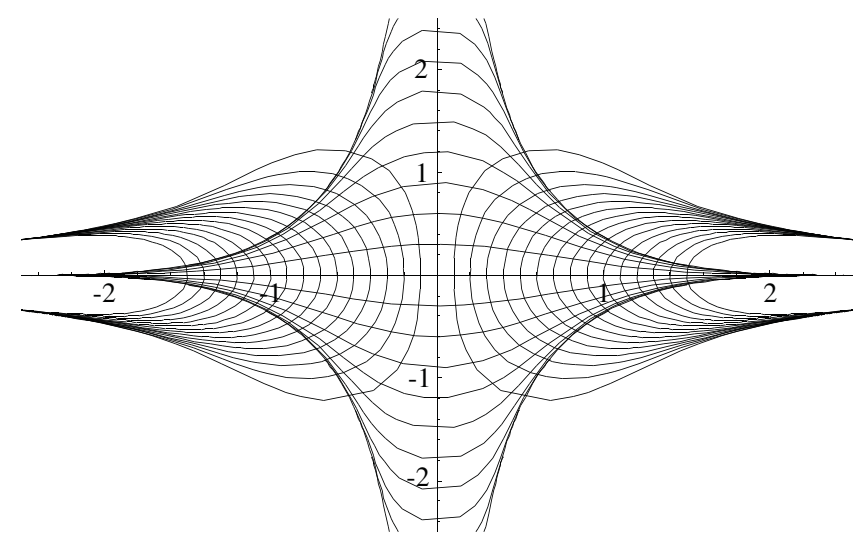

FIGURE 3. A grid of all geodesics parallel to the axes in the $\mathcal{g}$-plane corresponds to a small part of $\mathfrak{g}$.

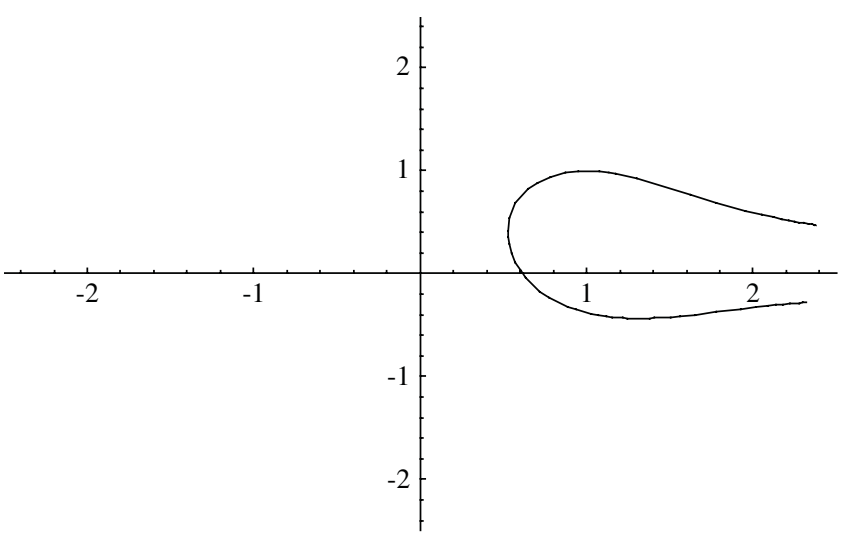

FIGURE 4. A Mathematica generated plot showing a geodesic in the g-plane passing through the point $(1,1)$ with slope 1 .

\section{The g-plane}

We now turn to the particular example of the plane with density $\exp \left(x^{2}-y^{2}\right)$, which we call the g-plane.

The change to Euclidean coordinates (Proposition 3.4) maps a grid of geodesics parallel to the axes, as seen in Figure 3, to straight parallel lines. However, this grid is not complete. Notice that the the $y$-axis reaches infinity at a weighted length of just $\sqrt{\pi} / 2$ from the origin, while all parallel geodesics have infinite weighted length. In Euclidean coordinates the surface is actually an infinitely sheeted Riemann surface with branch points on the $y$-axis at $y= \pm \sqrt{\pi} / 2$. Figures 4 and 5 show some other geodesics. 


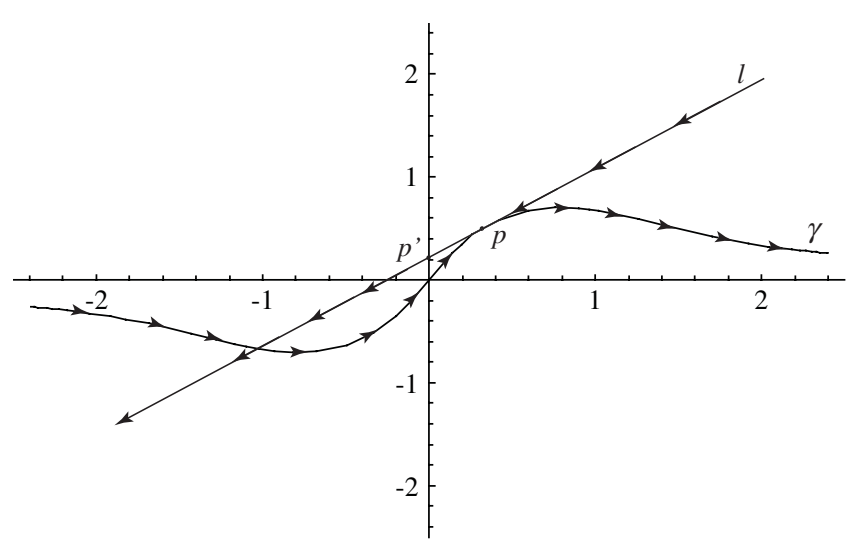

FIGURE 5. A Mathematica generated plot showing a geodesic in the g-plane passing through the origin illustrating the orientation of the line in Proposition 5.1.

PROPOSITION 5.1. Let $\gamma$ be a geodesic in $g$ and let $p$ be a point on $\gamma$ not on the $x$-axis or $y$-axis at which $\gamma$ is not tangent to a vertical line, as in Figure 5. Since the tangent line $l$ to $\gamma$ at $p$ is not vertical, it intersects the $y$-axis at some point $p^{\prime}$. We orient $l$ choosing the positive direction to go from $p$ to $p^{\prime}$. We give $\gamma$ the opposite orientation by comparison at $p$. Then, following $\gamma$ in positive direction (the direction pointed away from the $y$-axis at $p), \gamma$ converges to a line parallel to the $x$-axis.

PROOF. Without loss of generality, we assume $p$ is in the first quadrant, since the quadrants are symmetric. Let $\gamma$ be parameterized in terms of arc length, such that $\gamma(0)=(x(0), y(0))=p$, and such that $x^{\prime}(0)>0$. We have two cases: (i) $y^{\prime}(0)<0$ or (ii) $y^{\prime}(0) \geq 0$. Assume that $y^{\prime}(0)<0$. Also assume that $y(t) \neq 0$ for $t>0$. Now, we claim that $x^{\prime}(t) \geq 0$ and $y^{\prime}(t) \leq 0$ for all $t>0$. For this not to be the case, $\gamma$ would have to become vertical or horizontal at some point $t_{0}>0$ where $\gamma\left(t_{0}\right)$ is still in the first quadrant. We shall show that if $\gamma$ becomes vertical or horizontal at some point then curvature would point in the correct direction to make $x^{\prime}(t) \geq 0$ and $y^{\prime}(t) \leq 0$ again. Assume that $\gamma$ is horizontal at $t_{0}$. Then the normal vector $\mathbf{n}$ is vertical. So by the geodesic equation,

$$
\mathbf{n} \cdot(\boldsymbol{\kappa}-\nabla \varphi)=\mathbf{0}
$$

$(\boldsymbol{\kappa}-\nabla \varphi)$ must be horizontal. We know that $\boldsymbol{\kappa}$ is a multiple of $\mathbf{n}$, so it is vertical. Thus, it must exactly cancel the vertical component of $-\nabla \varphi$. In the first quadrant, $-\nabla \varphi=(-2 x, 2 y)$ has positive $y$-component, so to cancel, the curvature $\boldsymbol{\kappa}$ is vertical down. Thus, $y^{\prime}(t) \leq 0$ for $t>0$. Now, assume $\gamma$ is vertical at some point $t_{0}>0$. Then the normal vector $\mathbf{n}$ is horizontal, so $(\boldsymbol{\kappa}-\nabla \varphi)$ is vertical. Thus, $\boldsymbol{\kappa}$ must cancel the $x$-component of $-\nabla \varphi=(-2 x, 2 y)$ which is negative. So the curvature $\kappa$ is a vector in the positive $x$ direction, thus $x^{\prime}(t) \geq 0$ for $t>0$. With the conditions, $y^{\prime}(t) \leq 0$, $x^{\prime}(t) \geq 0$, and $y(t) \neq 0$ for $t>0$, we know that $\gamma$ must converge to a line parallel to 
the $x$-axis. We shall deal with what happens when $y(t)=0$ for some $t>0$ after we have dealt with the second case.

Moving to the second case, take $y^{\prime}(0) \geq 0$. We have already dispensed with the case $y^{\prime}(0)=0$. Since the curvature vector would point down, this becomes the first case instantaneously. Now, we already know what happens if the tangent vector becomes horizontal or vertical. If it becomes vertical, then we return to our original situation of $x^{\prime}(t) \geq 0$ and $y^{\prime}(0) \leq 0$. If it becomes horizontal, then we enter the first case. So what we want to show is that eventually the tangent vector becomes horizontal. To show this, it suffices to look at the magnitude and direction of the curvature vector. As noted, we know that $-\nabla \varphi=(-2 x, 2 y)=(a, b)$ has $a<0$ and $b>0$. Let $\mathbf{n}=(e, f)$, so $e \geq 0$ and $f<0$. We compute $\kappa=\mathbf{n} \cdot \nabla \varphi=-e a-f b$ where $\kappa=\kappa \mathbf{n}$. Since $-e a-f b>0$, the curvature is in the same direction as $\mathbf{n}$ which has a nonpositive vertical component. Thus, the geodesic is curving in the desired direction. However, we must show that it curves sufficiently quickly. Since $e \geq 0$ and $a<0,-e a>0$, so $\kappa=-e a-f b>-f b$. As $t$ increases, $f$ decreases to -1 and $b$ increases. Set $f_{\max }$ equal to its value at $t=0$ and define $b_{\min }$ analogously. Then $-f b>-f_{\max } b_{\min }$. Thus, by comparison with a constant curvature curve, we know that the curve eventually does become horizontal. This reduces case (ii) to case (i).

To finish the proof, we return to case (i) and remove the assumption that $y(t) \neq 0$ for $t>0$. Suppose that $y(t)=0$ for some $t_{0}>0$. By symmetry we can apply case (ii) to show that $\gamma$ then becomes horizontal and curves back towards the $x$-axis. Applying case (i), $\gamma$ must either approach a line parallel to the $x$-axis or cross the $x$-axis again. It cannot cross again, however, because it would violate uniqueness.

From Proposition 5.1, we get the following proposition which incompletely characterizes the end behaviour of geodesics.

PROPOSITION 5.2. If $\gamma$ is a geodesic on $\mathrm{g}$ that is not the $y$-axis then $\gamma$ converges to a line parallel to the $x$-axis in at least one direction. If $\gamma$ crosses the $x$-axis, then $\gamma$ converges to lines parallel to the $x$-axis in both directions.

PROOF. If $\gamma$ is the $x$-axis, then the proposition is clearly true, so we assume that this is not the case. Then, we know that a point which is not on one of the axes can be found. Applying Proposition 5.1 at this point to $\gamma$, we find that $\gamma$ converges to a line parallel to the $x$-axis in at least one direction. If $\gamma$ crosses the $x$-axis, then a point can be found on either side of the $x$-axis. Applying Proposition 5.1 to each of these points tells us that $\gamma$ converges to lines parallel to the $x$-axis in both directions.

In the remaining case, we believe two things are possible. At one end $\gamma$ converges to a line parallel to the $x$-axis. Either the other end curves around 180 degrees and converges to a line parallel to the $x$-axis, or it converges to a line parallel to the $y$-axis.

Despite the fact that Proposition 5.2 is an incomplete characterization, we can use it to prove a couple of useful corollaries.

COROLlARY 5.3. All geodesics in gexcept for the y-axis have infinite length. 


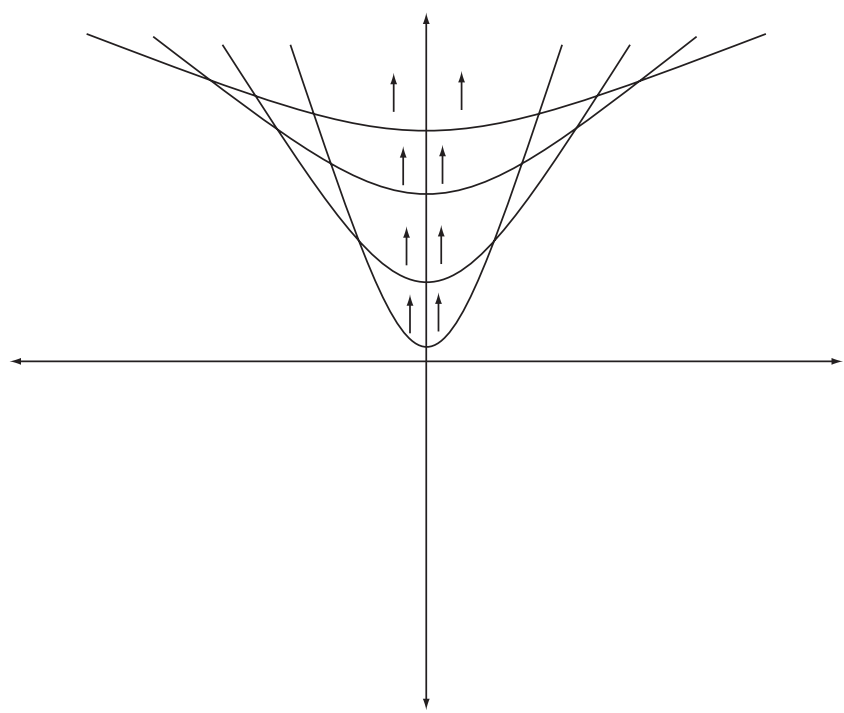

FIGURE 6. A sequence of regions in $\mathcal{g}$ which we conjecture approach the infimum perimeter for given area.

PROOF. This is a trivial corollary of Proposition 5.2. The quarters of plane on the left and right bounded by the lines $y=x$ and $y=-x$ have density greater than 1 , so any line converging to the $x$-axis has an infinite Euclidean length in this region and thus an infinite length.

Since an isoperimetric region minimizes perimeter and geodesics have infinite length, we have the following corollary.

COROLlARY 5.4. No isoperimetric region in $\mathrm{g}$ is bounded by a geodesic.

CONJeCture 5.5. There does not exist an isoperimetric region in $\mathrm{g}$.

We conjecture that $g$ is similar to the plane with density $e^{x}$, in that we can construct a sequence of regions (as in Figure 6) that approach the infimum perimeter for given area.

\section{Acknowledgements}

We would like to thank our advisor Frank Morgan for his patient and thoughtful help and input. We thank Robert Bryant for the Appendix. We thank the SMALL summer mathematics undergraduate research program for giving us the opportunity to do this study.

\section{Appendix A. Unique geodesic in the Gauss plane (Robert Bryant)}

At the Geometry Festival 2006, Frank Morgan mentioned the conjecture of Corwin et al. [3, Conjecture 6.1] that in the Gauss plane (with density proportional to 
$\left.\exp \left(x^{2}+y^{2}\right)\right)$ there is a unique closed geodesic (a circle). Overnight Robert Bryant supplied the following proof.

Proposition A.1. There are no simple closed geodesics in the Gaussian plane besides the circle.

PROOF. In the general case we assign the plane a density $\exp \left(a-b\left(x^{2}+y^{2}\right)\right)$ (with $b \geq 0$ ). For our purposes, in fact, a conformal change of metric to

$$
g=\exp \left(a-b\left(x^{2}+y^{2}\right)\right)\left(d x^{2}+d y^{2}\right)
$$

will suffice since we are looking at geodesics. This may be reduced by scaling to

$$
g=\exp \left(1-r^{2}\right)\left(d r^{2}+r^{2} d \theta^{2}\right) .
$$

(This makes the unit circle $(r=1)$ have length $2 \pi$ and be a geodesic.) We can write this as

$$
g=d s^{2}+f(s)^{2} d \theta^{2}
$$

with

$$
d s=\exp \left(\frac{1-r^{2}}{2}\right) d r
$$

and

$$
f(s)=r \exp \left(\frac{1-r^{2}}{2}\right) .
$$

Then

$$
f^{\prime}(s)=\frac{d f}{d s}=1-r^{2}
$$

and

$$
f^{\prime \prime}(s)=\frac{d f^{\prime}}{d s}=-2 r \exp \left(\frac{r^{2}-1}{2}\right),
$$

so the curvature $\kappa$ satisfies

$$
\kappa=\frac{-f^{\prime \prime}}{f}=2 \exp \left(r^{2}-1\right)>0 .
$$

It follows that $r=1$ is a geodesic of length $2 \pi$ and that conjugate points along this geodesic are separated by a distance of $\pi / \sqrt{k}=\pi / \sqrt{2}$.

Any unit speed geodesic satisfies

$$
\exp \left(1-r^{2}\right)\left(\dot{r}^{2}+r^{2} \dot{\theta}^{2}\right)=1
$$

and Clairaut's integral tells us that

$$
r^{2} \exp \left(1-r^{2}\right) \dot{\theta}=\alpha
$$




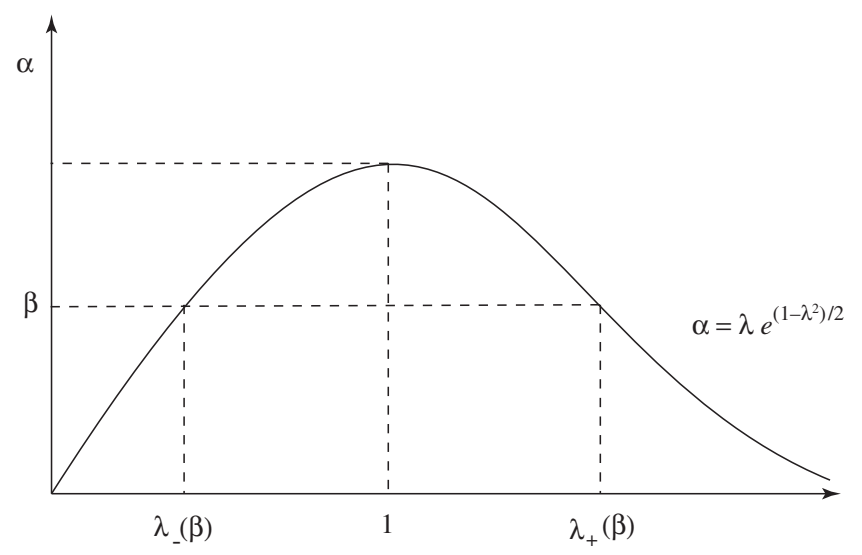

FIGURE A.1.

where $\alpha$ is a constant. The case $\alpha=0$ just gives the radial geodesics, which can be discarded. By reflectional symmetry $(\theta \rightarrow-\theta)$, assume that $\alpha>0$. Then $\theta$ is strictly increasing along a geodesic and hence can be used as a coordinate. The above equations then give

$$
\left(\frac{d r}{d \theta}\right)^{2}=\frac{r^{2}\left(\exp \left(1-r^{2}\right) r^{2}-\alpha^{2}\right)}{\alpha^{2}} .
$$

It follows from inspection that $\alpha \leq 1$, with $\alpha=1$ only for $r \equiv 1$, the closed circle. When $0<\alpha<1$, let $\lambda_{ \pm}(\alpha)$ be defined so that $0<\lambda_{-}(\alpha)<1<\lambda_{+}(\alpha)$ and

$$
\lambda_{ \pm}(\alpha)^{2} \exp \left(1-\lambda_{ \pm}(\alpha)^{2}\right)=\alpha^{2}
$$

as in Figure A.1.

It follows that a geodesic with $r^{2} \exp \left(1-r^{2}\right) \dot{\theta}=\alpha$ lies in the annulus

$$
\lambda_{-}(\alpha) \leq r \leq \lambda_{+}(\alpha)
$$

and is tangent to both boundary circles. The $\theta$ phase shift that the geodesic undergoes in travelling from the inner tangency to the outer tangency is then given by

$$
P(\alpha)=\int_{\lambda_{-}(\alpha)}^{\lambda_{+}(\alpha)} \frac{\alpha d r}{r \sqrt{r^{2} \exp \left(1-r^{2}\right)-\alpha^{2}}}
$$

with $0<\alpha<1$.

When $P(\alpha)=(p / q) \pi$ where $p / q$ is rational in lowest terms, the geodesic will close after touching the inner radius $r=\lambda_{-}(\alpha) q$ times and will wind $p$ times around the origin in order to close. In particular, it will be a simple closed geodesic only if $p=1$, that is, $P(\alpha)=\pi / q$ for some integer $q \geq 1$. 
By the conjugacy calculation, we know that

$$
\lim _{\alpha \rightarrow 1^{-}} P(\alpha)=\frac{\pi}{\sqrt{2}},
$$

so no geodesics that stay near $r=1$ can be simple closed geodesics.

Because the function $a(r)=r \exp \left(\left(1-r^{2}\right) / 2\right)$ satisfies $0<a(r) \leq 1$ for $0<r<1$ and $a^{\prime}$ vanishes only at $r=1$ while $a^{\prime \prime}(1)<0$, it follows that there is a unique smooth function $\rho:(0, \infty) \rightarrow(-\pi / 2, \pi / 2)$ such that $\cos \rho=r \exp \left(\left(1-r^{2}\right) / 2\right)$ and such that $d \rho / d r>0$. (In fact, $\rho$ extends smoothly to $\rho:(-\infty, \infty) \rightarrow(-3 \pi / 2, \pi / 2)$ by requiring that $\rho(-x)=-\pi-\rho(x)$ for $x \geq 0$. However, this is not likely to be useful.)

Setting $\alpha=\cos \phi$ for $0<\phi<\pi / 2$, the formula for $P$ can be written as

$$
P(\cos \phi)=\int_{-\phi}^{\phi} \frac{\cos (\phi) h(\rho) d \rho}{\cos (\rho) \sqrt{\cos ^{2}(\rho)-\cos ^{2}(\phi)}},
$$

where $h$ is the unique function of $(-\pi / 2, \pi / 2)$ such that

$$
\frac{h(\rho) d \rho}{\cos \rho}=\frac{d r}{r}
$$

that is,

$$
h(\rho) d \rho=\exp \left(\frac{1-r^{2}}{2}\right) d r
$$

Since

$$
-\sin (\rho) d \rho=\left(1-r^{2}\right) \exp \left(\frac{1-r^{2}}{2}\right) d r=\left(1-r^{2}\right) h(\rho) d \rho,
$$

it follows that

$$
h(\rho)=\frac{\sin (\rho)}{r^{2}-1}
$$

so

$$
h(\rho)^{2}=\frac{1-\cos ^{2} \rho}{\left(1-r^{2}\right)^{2}}=\frac{1-r^{2} \exp \left(1-r^{2}\right)}{\left(1-r^{2}\right)^{2}} .
$$

It follows that $h(-\pi / 2)=1$, and that $h(\pi / 2)=0$. We also see that $h(0)=1 / \sqrt{2}$.

In fact, it is straightforward to compute that $h^{\prime \prime} \leq 0$ on $(-\pi / 2, \pi / 2)$ and that the graph looks like Figure A.2 (see Note A.2 below). In particular, note that $h(\rho)>(\pi / 2-\rho) / \pi=1 / 2-\rho / \pi$. So we compute

$$
\begin{aligned}
P(\cos (\phi)) & >\int_{-\phi}^{\phi} \frac{\cos (\phi)((1 / 2)-(\rho / \pi))}{\cos (\rho) \sqrt{\cos ^{2}(\rho)-\cos ^{2}(\phi)}} d \rho \\
& =\int_{-\phi}^{\phi} \frac{(1 / 2) \cos (\phi)}{\cos (\rho) \sqrt{\cos ^{2}(\rho)-\cos ^{2}(\phi)}} d \rho \\
& =\frac{\pi}{2} .
\end{aligned}
$$

The integral can be computed using basic calculus but is tricky (see Note A.3). 


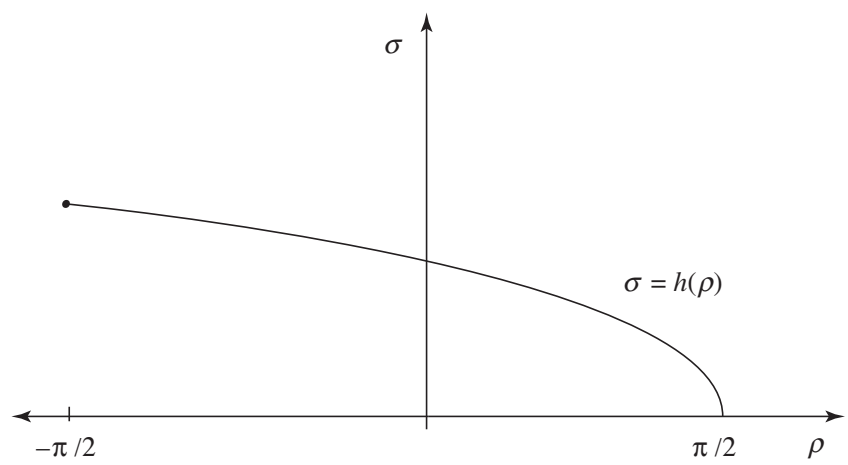

FigURE A.2.

Then $h(\rho) \equiv 1$, so the integral equals $\pi$ because any two geodesics on the unit sphere intersect at antipodal points and are thus separated by a distance of exactly $\pi$.

Thus $P(\cos (\phi))>\pi / 2$ for $0<\phi<\pi / 2$. Note also that $h(\rho)<1$ except when $\rho=-\pi / 2$, so for $0<\phi<\pi / 2, P(\cos (\phi))<2 E(\cos (\theta))=\pi$, twice the value of the integral. In particular, we have $\pi / 2<P(\alpha)<\pi$ for all $0<\alpha<1$. Thus, there are no other simple closed geodesics.

Note A.2. Simple calculus suffices to show that $h^{\prime \prime} \leq 0$ on $(-\pi / 2, \pi / 2)$. We compute that $h^{\prime \prime}$ is the positive function $(1-R)^{-6} \exp ((1-R) / 2)$ times

$$
g(R)=-1-4 R-6 R^{2}-R^{4}+\left(2+12 R-2 R^{2}\right) \exp (R-1)
$$

where $R=r^{2}$. So it is sufficient to show $g(R) \leq 0$ for $R \geq 0$. We divide the region into nine intervals and prove this for each one. The intervals are [0, 1.24504], $[1.24504,1.24505],[1.24505,2.129],[2.129,2.13],[2.13,2.17],[2.17,2.171]$, $[2.171,2.7794],[2.7794,2.781],[2.781, \infty)$.

We now look at when $g^{\prime \prime}(R)$ is positive. We compute

$$
g^{\prime \prime}(R)=-12-12 R^{2}+\left(22+4 R-2 R^{2}\right) \exp (R-1) \leq 0
$$

if and only if

$$
\exp (R-1) \leq \frac{12+12 R^{2}}{22+4 R-2 R^{2}},
$$

which is true if and only if

$$
R-1 \leq \ln \left(12+12 R^{2}\right)-\ln \left(22+4 R-2 R^{2}\right) .
$$

Setting

$$
m(R)=\ln \left(12+12 R^{2}\right)-\ln \left(22+4 R-2 R^{2}\right)-R+1
$$


we compute

$$
m^{\prime \prime}(R)=\frac{4\left(67+21 R-56 R^{2}-26 R^{3}+17 R^{4}+R^{5}\right)}{\left(1+R^{2}\right)^{2}\left(-11-2 R+R^{2}\right)^{2}},
$$

the positivity of which is determined by the numerator. Of course, this function is only meaningful up until $1+2 \sqrt{(} 3) \approx 4.464$ when $\ln \left(22+4 R-2 R^{2}\right)$ becomes undefined. Since this is a fifth-degree polynomial we can trust our numerical estimation of the roots. These are approximately (rounding final digit) $\{1.2450449,2.12908,-1.060+0.510 i,-18.254,-1.060-0.510 i\} . \quad$ The two roots which fall in our range are 1.2450449 and 2.12908 .

Testing values shows that between 0 and 1.24504 the concavity $m^{\prime \prime}(R)$ is positive. At $R=1, m(R)=m^{\prime}(R)=0$, so on this range $m(R) \geq 0$, so $g^{\prime \prime} \leq 0$. Since $g(1)=$ $g^{\prime}(1)=0$, this suffices to show that $g(R) \leq 0$ on the first interval.

For the second interval, [1.245 04, 1.24505$]$, we overestimate $g(R)$ by noting that it has the form $p_{1}(R)+p_{2}(R) \exp (R-1)$. Since $p_{1}(R)$ is always negative and $p_{2}(R)$ is positive for $3-\sqrt{10}<R<3+\sqrt{10}$, we overestimate by setting $\exp (R-1)$ to its max value on the range - in this case, $\exp (0.24505)$. This gives a fourthdegree polynomial $p(R)=p_{1}(R)+p_{2}(R) \exp (0.24505)$. It is easy to characterize the positive and negative intervals of this polynomial by solving for the roots. In this case, we find $0>p(R) \geq g(R)$ over the second interval.

For the third interval, [1.24505, 2.129], we note that $m^{\prime \prime}(R)$ is negative. So it suffices to check the values at the endpoints to show $m(R)>0$. These are $m(1.24505)>0.0029$ and $m(2.129)>0.0008$. Thus $m(R)>0$ over this interval, so $g^{\prime \prime}$ is negative. Since $g(1.24505)<-0.0005$ and $g^{\prime}(1.24505)<-0.008$, this tangent line gives us that $g(R)<0$ on this interval.

The fourth interval, [2.129,2.13], is similar to the second interval. We have $0>p(R)=p_{1}(R)+p_{2}(R) \exp (1.13) \geq g(R)$.

For the fifth interval, $[2.13,2.17], m^{\prime \prime}(R)>0$, so $m$ is bounded below on this interval by the tangent line at 2.17. Since the slope is $m^{\prime}(2.17)<-0.01967$, the lowest point reached is at $m(2.17)>0.000002$. So $m(R)>0$, which implies that $g^{\prime \prime}(R)<0$. Since $g(2.13)<-0.09$ and $g^{\prime}(2.13)<-0.2$, this tangent line shows that $g(R)<0$ for this interval.

The sixth interval, [2.17, 2.171], is again similar to the second. In this case $0>p(R)=p_{1}(R)+p_{2}(R) \exp (1.171) \geq g(R)$.

For the seventh interval, [2.171, 2.7794], $m^{\prime \prime}(R)>0$, so the secant line between $m(2.171)<-0.000017$ and $m(2.7794)<-0.000018$ shows that $m(R)<0$. Thus $g^{\prime \prime}(R)>0$. So the secant line between $g(2.171)<-0.1$ and $g(2.7794)<-0.1$ shows that $g(R)<0$.

The eighth interval, [2.7794, 2.781], is similar to the second. In this case $0>$ $p(R)=p_{1}(R)+p_{2}(R) \exp (1.781)>g(R)$.

For the ninth interval, [2.781, $\infty)$, we look at $g^{(4)}=-24+(26-4 R-$ $\left.2 R^{2}\right) \exp (R-1)$. Since, for $R \geq 2.742>\sqrt{14}-1,\left(26-4 R-2 R^{2}\right)<0$, then 
$g^{(4)}(R)<0$. Now, $g^{(3)}(2.781)<-4.2, g^{\prime \prime}(2.781)<-0.004, g^{\prime}(2.781)<-0.05$, and $g(2.781)<-0.19$, so $g(R)<0$ for all $R \geq 2.781$.

Note A.3. The integral is computed as follows:

$$
\begin{aligned}
P(\cos (\phi)) & >\int_{-\phi}^{\phi} \frac{(1 / 2) \cos (\phi)}{\cos (\rho) \sqrt{\cos ^{2}(\rho)-\cos ^{2}(\phi)}} d \rho \\
& =\int_{0}^{\phi} \frac{\cos (\phi)}{\cos (\rho) \sqrt{\cos ^{2}(\rho)-\cos ^{2}(\phi)}} d \rho \\
& =\int_{1}^{c} \frac{-c}{u \sqrt{u^{2}-c^{2}} \sqrt{1-u^{2}}} d u
\end{aligned}
$$

where $c=\cos (\phi)$ and $u=\cos (\rho)$. Then substituting $v=u^{2}$ gives

$$
\begin{aligned}
& =\frac{c}{2} \int_{c}^{1} \frac{1}{u^{2} \sqrt{u^{2}-c^{2}} \sqrt{1-u^{2}}} 2 u d u \\
& =\frac{c}{2} \int_{c^{2}}^{1} \frac{1}{v \sqrt{\left(v-c^{2}\right)(1-v)}} d v \\
& =\frac{c}{2} \int_{c^{2}}^{1} \frac{1}{v \sqrt{\left(\left(c^{2}-1\right) / 2\right)^{2}-\left(v-\left(1+c^{2}\right) / 2\right)^{2}}} d v .
\end{aligned}
$$

Now, we substitute $v=\left(c^{2}-1\right) / 2 \sin (\theta)+\left(1+c^{2}\right) / 2$ and get

$$
\frac{c}{2} \int_{-\pi / 2}^{\pi / 2} \frac{\left(c^{2}-1\right) / 2 \cos (\theta)}{\left(\left(c^{2}-1\right) / 2 \sin (\theta)+\left(\left(1+c^{2}\right) / 2\right)\right)\left(c^{2}-1\right) / 2 \cos (\theta)} d \theta
$$

which equals

$$
\frac{c}{2} \int_{-\pi / 2}^{\pi / 2} \frac{1}{\left(c^{2}-1\right) / 2 \sin (\theta)+\left(\left(1+c^{2}\right) / 2\right)} d \theta .
$$

So the integral can be evaluated as

$$
\begin{aligned}
\frac{c}{2}\left[\frac{2}{c} \tan ^{-1}\left(\frac{\tan (\theta / 2)\left(1+c^{2}\right)-1+c^{2}}{2 c}\right)\right]_{-\pi / 2}^{\pi / 2} & =\tan ^{-1} c+\tan ^{-1}\left(\frac{1}{c}\right) \\
& =\frac{\pi}{2} .
\end{aligned}
$$

Another way to attain this equality is to do the calculation for $P$ for the round metric on the unit sphere. 


\section{References}

[1] A. L. Besse, Einstein Manifolds (Springer, Berlin, 1987).

[2] H. Cohn, Conformal Mapping on Riemann Surfaces (McGraw-Hill, New York, 1967).

[3] I. Corwin, N. Hoffman, S. Hurder, V. Šešum and Y. Xu, 'Differential geometry of manifolds with density', Rose-Hulman Und. Math. J. 7(1) (2006).

[4] F. Morgan, Riemannian Geometry: A Beginner's Guide, 2nd edn (A K Peters, Wellesley, MA, 1998).

[5] - Geometric Measure Theory, 4th edn (Academic Press, San Diego, 2008).

[6] _ 'Regularity of isoperimetric hypersurfaces in Riemannian manifolds', Trans. Amer. Math. Soc. 52(12) (2003), 5041-5052.

[7] _ _ 'Manifolds with density', Notices Amer. Math. Soc. 52 (2005), 853-858.

[8] _ 'Myers' theorem with density', Kodai Math. J. 29 (2006), 454-460.

[9] C. Rosales, A. Cañete, V. Bayle and F. Morgan, Calc. Varn. Partial Differential Equations 31 (2008), $27-46$.

COLIN CARROLL, Department of Mathematics and Statistics, Williams College, Williamstown, MA 01267, USA

e-mail: 07cdc@williams.edu

ADAM JACOB, Department of Mathematics, University of California, Berkeley, CA 94720, USA

e-mail: ajacob@math.columbia.edu

CONOR QUINN, Department of Mathematics and Statistics, Williams College, Williamstown, MA 01267, USA

e-mail:07cbq@williams.edu

ROBIN WALTERS, Department of Mathematics, Harvard University, Cambridge, MA 02138, USA

e-mail: rwalters@post.harvard.edu 